\title{
Mesenchymal Chondrosarcoma, Clinicopathological Characteristics of an Uncommon Tumor
}

\author{
Muhammad Raza ${ }^{1}$, Mudassar Hussain ${ }^{1}$, Nasir Uddin ${ }^{2}$, Noreen Akhter ${ }^{1}$ and Usman Hassan ${ }^{1^{*}}$ \\ ${ }^{1}$ Department of Pathology, Shaukat Khanum Memorial Cancer Hospital and Research Centre, Lahore, Pakistan \\ ${ }^{2}$ Consultant Histopathologist, Agha Khan University Hospital, Karachi, Pakistan
}

"Corresponding author: Hassan U, Consultant Histopathologist, Department of Pathology, Shaukat Khanum Memorial Cancer Hospital and Research Centre, Lahore, Pakistan, Tel: +923408400058; E-mail: drusmanhassan256@gmail.com

Received date: May 13, 2018; Accepted date: May 25, 2018; Published date: May 31, 2018

Copyright: (c) 2018 Raza M, et al. This is an open-access article distributed under the terms of the Creative Commons Attribution License, which permits unrestricted use, distribution, and reproduction in any medium, provided the original author and source are credited.

\begin{abstract}
Mesenchymal chondrosarcomas (MCS) are very rare malignant tumors. They comprise $2-10 \%$ of all chondrosarcomas. Approximately 600 cases of MCS have been published, as case reports and small series. In English language medical literature only three series include 20 or more cases have been described. This paper aims to document various histological findings, histological patterns that can be seen in MCS. In addition, demographic features, clinical and radiological findings and survival data are also incorporated to assess prognostic impact of various histological findings.
\end{abstract}

This is a descriptive cross sectional study including cases of MCS diagnosed at Shaukat Khanum Memorial Cancer Hospital and Research Center, Lahore, Pakistan between 2002 and 2012. The slides were reviewed by two consultant pathologists independently and findings (pattern, cellularity, spindle cell component, necrosis, mitoses, hemangiopericytoma like vessels) were documented on a specially developed form. The clinical information was extracted from case files. Survival data was obtained by contacting the patient or their families in those cases for which contact numbers were available. Data analysis was done using SPSS 20.0 .

Of the total 18 cases, 10 were males $(55.6 \%)$ and 8 were females (44.4\%). Patients were most commonly in their second or third decades of life. Tumor locations included craniofacial bones $(n=5,28 \%)$, ribs and chest wall $(n=3$, $16 \%)$, spine $(n=3,16 \%)$, and the lower extremity bones $(n=5,28 \%)$. Extraskeletal sites included parapharyngeal region $(n=1,6 \%)$ and thigh $(n=1,6 \%)$. Histologically biphasic pattern was seen 16 cases $(89 \%)$. Two cases showed sheets of round to spindle cells with very focal cartilaginous component $(11 \%)$. Spindle cell component was seen in 14 cases. Necrosis was present in 7 cases (38.9\%). Survival data was available for 11 patients (61\%). Among these, $n=7(64 \%)$ patients developed metastatic disease. The mean survival for cases with metastatic disease was 14.2 months. In patients without metastatic disease, mean survival was 37 months.

As the incidence of mesenchymal chondrosarcoma is extremely low, the limited number of patients discussed in most of the studies precludes statistically significant conclusions. MCS should be considered in the differential diagnosis, when pathologists encounter a bone and soft tissue tumor with biphasic pattern composed of round to spindle cell component and hyaline cartilage. Given the diagnostic pitfalls discussed, clinicians may consider obtaining larger biopsy specimens to limit sampling error or confirming the diagnosis with immunohistochemical stains or genetic analysis when a larger biopsy is not possible.

Keywords: Mesenchymal chondrosarcoma; Biphasic pattern; Immunohistochemistry; Pathological

\section{Introduction}

Mesenchymal chondrosarcomas (MCS) are very rare malignant tumors, described first by Lichtenstein and Bernstein in 1959 [1]. They comprise $2-10 \%$ of all chondrosarcomas [2-7].

Both skeletal and extra-skeletal sites can be involved. The preferred sites include axial skeleton and femur [1-4]. Extra-skeletal cases arise in soft tissue at a variety of sites and sometimes show visceral involvement [8-11].

Patients often present with swelling, pain and in few cases with pathological fracture. Radiological investigations reveal an aggressive lytic and destructive appearance. Extension into adjacent soft tissue is also commonly seen. Many cases show calcifications as well [7].

Histological sections show a unique biphasic pattern of variable amounts of sheets of small round blue cells, sharply demarcated from lobule of hyaline cartilage [1]. The diagnosis is usually straight forward in resection samples however small biopsies can pose a diagnostic challenge due to the frequently seen crushing artifacts or if the biopsy fails to sample cartilaginous areas. In such instances, positive SOX9 staining $[12,13]$ and detection of HEY1-NCOA2 fusion can be used as reliable diagnostic adjuncts[14].

Mesenchymal chondrosarcomas are ideally treated with a combination of surgery, chemotherapy and radiotherapy [15]. However, these tumors have a high local recurrence rate [16,17]. Metastasis can be seen after a long disease free interval. Therefore, the overall prognosis is poor with 10 year survival rates ranging from 
Citation: Raza M, Hussain M, Uddin N, Akhter N, Hassan U (2018) Mesenchymal Chondrosarcoma, Clinicopathological Characteristics of an Uncommon Tumor. J Cytol Histol 9: 505. doi:10.4172/2157-7099.1000505

Page 2 of 5

21-67\% [15-18]. This figure greatly varies depending upon the site, surgical resectability, type and duration of given therapy.

Approximately 600 cases of MCS have been published, as case reports and small series. In English language medical literature only three series include 20 or more cases have been described. This paper aims to document various histological findings, histological patterns that can be seen in MCS. In addition, demographic features, clinical and radiological findings and survival data are also incorporated to assess prognostic impact of various histological findings.

\section{Material and Methods}

This is a descriptive cross sectional study including cases of MCS diagnosed at Shaukat Khanum Memorial Cancer Hospital and Research Center, Lahore, Pakistan between 2002 and 2012. Cases with history of neoadjuvant therapy and with incomplete clinical history were excluded. Slides were pulled out along with case files. The slides were reviewed by two consultant pathologists independently and findings (pattern, cellularity, spindle cell component, necrosis, mitoses, and hemangiopericytoma like vessels) were documented on a specially developed form.

The cases with discrepant data were reviewed by a senior consultant before the findings were finalized. The clinical information was extracted from case files. Number of blocks made was also recorded. Staining method used in our laboratory for light microscopy is hematoxylin and eosin which is done through automated staining machine of Leica Biosystems. For immunohistochemical stains our lab use automated immunostainer provided by Leica Biosystems (Leica BOND) and all procedures are followed as indicated in instruction manuals of Leica Bond immunostainer and immunohistochemistry antibody clone literature. CD99 immunohistochemical antibody was used only in two cases in this study (NCL-L-CD99-187-CD99 Novocastra). Survival data was obtained by contacting the patient or their families in those cases for which contact numbers were available. Data analysis was done using SPSS 20.0.

\section{Results}

Of the total 18 patients, 10 were of male gender $(55.6 \%)$ and 8 were of female gender $(44.4 \%)$. Age ranged from 10 to 50 years (mean age, 27.8 years; median age, 28 years). Male patients were younger at presentation than female patients (mean age, males, 23.9 years; mean age, females, 29.6 years). Patients were most commonly in their second or third decades of life. Most common presenting complaint was mass ( 9 cases), followed by pain ( 5 cases) and 4 patients had both mass and pain. Tumor locations included craniofacial bones $(n=5,28 \%)$, ribs and chest wall $(n=3,16 \%)$, spine $(n=3,16 \%)$, and the lower extremity bones $(n=5,28 \%)$. Extraskeletal sites included parapharyngeal region $(n=1$, $6 \%)$ and thigh $(n=1,6 \%)$. Out of these 18 patients, four belonged to pediatric age group with age ranging from 10 to 17 years and with male to female ratio 2:2. Two patients presented with mass and two with pain. Tumor location was craniofacial bones in 2 cases, spine in 1 case and lower extremity bone in 1 case. Out of the 14 adult cases, 8 were males and 6 were females. About 7 patients presented with mass, 3 with pain and 4 with both mass and pain. Three patients had tumor is craniofacial bones, 3 in rib and chest wall, 2 in spine and 4 in lower extremity bones. Extraskeletal sites included parapharyngeal region $(\mathrm{n}=1,6 \%)$ and thigh $(\mathrm{n}=1,6 \%)$.

Two out of 18 cases were incisional biopsies and 16 were open biopsies. Complete excision of the tumor was not received in any of the case. Both incisional biopsy cases were entirely submitted in one block each. Average size of the specimen received in cases received as open biopsies was $3.4 \mathrm{~cm}$ (ranging in size from $2 \mathrm{~cm}$ to $10 \mathrm{~cm}$ ). In each and every case, 1 block was made from every $\mathrm{cm}$ of the tumor. Therefore average number of blocks made per case was similar to the average size of the specimen received.

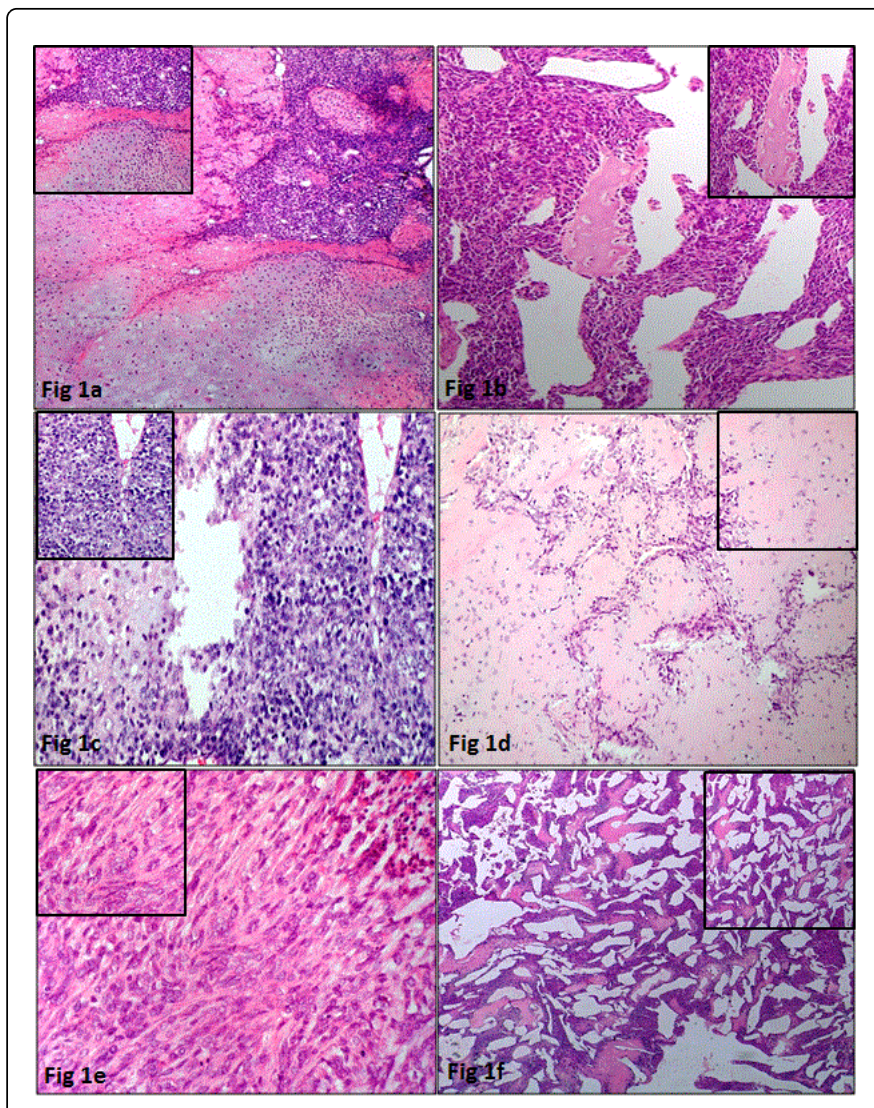

Figure 1: (a) Tumor showing biphasic pattern with round blue cell component juxtaposed with cartilaginous component $(\mathrm{H}$ and $\mathrm{E}$ stain $[20 \mathrm{x}]$ ). Inset is showing abrupt transition between round blue cell component and cartilage ( $\mathrm{H}$ and $\mathrm{E}$ stain [40x]). (b) Tumor composed of spindle cells with very focal cartilaginous component ( $\mathrm{H}$ and $\mathrm{E}$ stain $[20 \mathrm{x}]$ ). Inset is showing focal cartilage differentiation ( $\mathrm{H}$ and $\mathrm{E}$ stain $[40 \mathrm{x}])$. (c) Tumor with high cellularity (H and $\mathrm{E}$ stain $[20 \mathrm{x}]$ ). Inset is showing sheets of round blue cell component with high cellularity (H and E stain $[40 x]$ ). (d) Tumor with low cellularity with increased deposition of background matrix $(\mathrm{H}$ and $\mathrm{E}$ stain $[20 \mathrm{x}]$ ). Inset showing matrix deposition ( $\mathrm{H}$ and $\mathrm{E}$ stain $[40 \mathrm{x}])$. (e) Tumor composed of spindle cell component (H and $\mathrm{E}$ stain $[20 x])$. Inset showing sheets of spindle cells ( $\mathrm{H}$ and $\mathrm{E}$ stain $[40 \mathrm{x}])$. (f): Tumor composed of hemangiopericytoma like areas $(\mathrm{H}$ and $\mathrm{E}$ stain $[20 \mathrm{x}])$. Inset showing prominent vascular pattern in the form of hemangiopericytoma pattern ( $\mathrm{H}$ and $\mathrm{E}$ stain $[40 \mathrm{x}])$.

Histologically biphasic pattern was seen 16 cases including 3 pediatric cases and 13 adult cases (89\%) (Figure 1a). Two cases (one pediatric and one adult case) showed sheets of round to spindle cells with very focal cartilaginous component (11\%) (Figure 1b). Both cases were however, incisional biopsies. The cellularity was high in 16 cases (Figure 1c) whereas in 2 cases tumor cellularity was low due to 
increased deposition of background matrix (Figure 1d). Spindle cell component was seen in 14 cases (77.8\%) (Figure 1e). The percentage of this component ranged from $10 \%$ to $90 \%$ and $n=3$ tumors $(17 \%)$ demonstrated predominantly spindle cells, rather than round cells. Hemangiopericytoma like vessels were identified in 13 cases $(72.2 \%)$ (Figure 1f). Necrosis was present in 7 cases (38.9\%). The histological appearances therefore, varied from highly cellular round blue cell tumors with marked necrosis and rare cartilaginous islands to less cellular predominantly spindle cell neoplasms showing no necrosis and abundant cartilage. Immunohistochemical study was not performed in most of the cases as most of the tumors (89\%) showed biphasic pattern. In two cases, in which CD99 (NCL-L-CD99-187-CD99 Novocastra) was performed; it gave positive results (cytoplasmic and membranous staining in more than $50 \%$ of cells).

Survival data was available for 11 patients including 2 pediatric and 9 adult cases (61\%). Follow up period after diagnosis ranged from 1 month to 98 months with mean survival of 22.6 months. Among these, $\mathrm{n}=7$ (64\%) patients (including two pediatric patients) developed metastatic disease. Metastatic sites included lung $(n=5)$, brain $(n=1)$ and spine $(n=1)$. Of these 7 cases, $n=5$ died of disease between 1 and 39 months of presentation. The mean survival for cases with metastatic disease was 14.2 months. In patients without metastatic disease, mean survival was 37 months and none of these patients died of disease. Complete surgical resection of primary tumor was achieved in 6 cases (33\%).

In patients with metastatic and without metastatic disease, histological features were not different and no histological feature (pattern, cellularity, spindle cell component, necrosis, mitoses, hemangiopericytoma like vessels) was particularly found associated with bad and good prognosis (Table 1).

\begin{tabular}{|c|c|c|c|c|c|c|}
\hline Sr. no. & Source, year & No. of cases & M:F ratio & Age range/median & $\begin{array}{l}\text { Location Bone: } \\
\text { soft tissue }\end{array}$ & Metastasis \\
\hline 1 & Salvador, et al. [4], 1971 & 30 & $11: 19$ & $5-74 / 23.5$ & $21: 09$ & Sep-30 \\
\hline 2 & Huvos, et al .[2], 1983 & 35 & $20: 15$ & $6-70 / \mathrm{NR}^{*}$ & $30: 05: 00$ & $32 / 35$ \\
\hline 3 & Nakashima, et al. [3], 1986 & 109 & $52: 57: 00$ & $5-74 / N^{*}$ & $86: 39: 00$ & $25 / 109$ \\
\hline 4 & Cesari, et al. [15], 2007 & 26 & $10: 16$ & $20-71 / 31$ & $17: 09$ & $16 / 26$ \\
\hline 5 & Fanurg-Smith, et al..* [13], 2010 & 22 & $06: 16$ & $12-69 / 26.5$ & 17:09 & Jan-22 \\
\hline 6 & Shakked, et al. [7], 2012 & 20 & 09:11 & $7-37 / 23.5$ & $18: 02$ & Jun-20 \\
\hline
\end{tabular}

Table 1: Key features of previously published series.

\section{Discussion}

Mesenchymal chondrosarcoma is recognized for the last 58 years, however, largely due to its rarity, it continues to present diagnostic problems and management challenges [7]. Only few case series are published to date. Key features from these studies are summarized in Table 1. Radiographic images show an expansile lytic bony or soft tissue mass with local destruction and occasional fine calcifications [2]. Histologically these neoplasms are characterized by a unique, biphasic pattern, composed of sheets of undifferentiated mesenchymal, round or spindled cells along with islands of well differentiated hyaline cartilage. The round cell component often shows high cellularity, foci of necrosis, frequent mitoses and a hemangiopericytoma like vascular pattern. The cartilaginous areas may show calcification, ossification and osteoid production [6,7]. The diagnosis is straight forward if both components are sampled; however, the absence of cartilage in the biopsy sample can cause confusion with other small, round, blue-cell tumors e.g. Ewing's sarcoma; lymphoma; neuroblastoma; desmoplastic small round cell tumor; and small cell osteosarcoma. Typical immunohistochemistry findings include positivity of the mesenchymal portion for vimentin, Leu7, and CD99 and positivity of the cartilaginous regions for $\mathrm{S} 100$ protein $[6,19]$. Once again, absence of either component can lead to a skewed immunohistochemical picture. CD99, although immunoreactive in majority of the cases of mesenchymal chondrosarcoma, also shows diffuse strong expression in Ewing sarcoma and some expression in small cell variant of osteosarcoma further clouding the distinction between these tumors [20]. Wehrli et al compared immunohistochemistry profiles of 12 mesenchymal chondrosarcoma samples with many other small, round, blue-cell tumors and demonstrated expression of Sox9 in both cartilaginous and mesenchymal components in 21 out of 22 mesenchymal chondrosarcomas (95.5\%) [12]. Sox9 is a master regulator for cartilage development, and was not expressed in any of the 73 other round, blue-cell tumors evaluated by Wehrli et al. These findings were confirmed by another study conducted by FanburgSmith et al. [13] noting Sox9 expression in 21 of 22 cases of mesenchymal chondrosarcoma (95.5\%). In a recent study, FLI-1 was used for exclusion of mesenchymal chondrosarcoma and for identifying Ewing sarcoma when only small, round blue cells are available for study; 6 of 8 Ewing sarcoma samples (75\%) stained for FLI-1, and 0 of 10 mesenchymal chondrosarcoma samples $(0 \%)$ stained for FLI-1 [21,22]. Sox9 and FLI-1 may prove to be diagnostic aids, even in mesenchymal chondrosarcoma samples lacking cartilage islands. Cytogenetic investigations demonstrate findings in mesenchymal chondrosarcoma that are also seen in Ewing sarcoma including reciprocal translocation $(11 ; 22)$ (q24; q12) $[23,24]$. In our experience, immunohistochemistry is not essential for diagnosis in those cases in which one could find typical biphasic pattern with round blue cell component juxtaposed with cartilaginous component. In smaller biopsies, immunohistochemistry does have a role. 
Prognosis is variable with published 10 year overall survival rates ranging from $21 \%$ to $67 \%[15,18]$. Many patients live for long periods, even with metastatic disease, whereas some patients die shortly after diagnosis. This is further complicated by variations in adjuvant treatment protocols. Improved survival has been reported in patients who receive chemotherapy [15]. Current management emphasizes on both local and systemic control. Wide surgical resection is the mainstay of local treatment and patient undergoing complete surgical resection are reported to have a better survival.

Enhanced understanding of tumorigenesis after identification of the translocation $(11 ; 22)$ leading to fusion of HEY1-NCOA genes has opened avenues for further research and development of targeted therapies $[14,25,26]$. A study showed increased activity of the platelet derived growth factor receptor a (PDGFR-a) cell proliferation pathway, suggesting that therapies targeting this pathway may be useful in treating mesenchymal chondrosarcoma [27,28].

\section{Conclusion}

As the incidence of mesenchymal chondrosarcoma is extremely low, the limited number of patients discussed in most of the studies precludes statistically significant conclusions [29]. The demographic, clinical, histologic features and survival analysis in this study may contribute a little bit to the overall data of MCS. MCS should be suspected when pathologists encounter a bone and soft tissue tumor with biphasic pattern composed of round to spindle cell component and hyaline cartilage. Given the diagnostic pitfalls discussed, clinicians may consider obtaining larger biopsy specimens to limit sampling error or confirming the diagnosis with immunomarkers or genetic analysis when a larger biopsy is not possible.

\section{References}

1. Lichtenstein L, Bernstein D (1959) Unusual benign and malignant chondroid tumors of bone: a survey of some mesenchymal cartilage tumors and malignant chondroblastic tumors, including a few multicentric ones, as well as many atypical benign chondroblastomas and chondromyxoid fibromas. Cancer 12: 1142-1157.

2. Huvos AG, Rosen G, Dabska M, Marcove RC (1983) Mesenchymal chondrosarcoma: a clinicopathologic analysis of 35 patients with emphasis on treatment. Cancer 51: 1230-1237.

3. Nakashima Y, Unni KK, Shives TC, Swee RG, Dahlin DC (1986) Mesenchymal chondrosarcoma of bone and soft tissue: a review of 111 cases. Cancer 57: 2444-2453.

4. Salvador AH, Beabout JW, Dahlin DC (1971) Mesenchymal chondrosarcoma - observations on 30 new cases. Cancer 28: 605-615.

5. Bertoni F, Picci P, Bacchini P (1983) Mesenchymal chondrosarcoma of bone and soft tissues. Cancer 52: 533-541.

6. Nakashima Y, Park YK, Sugano O (2002) Mesenchymal chondrosarcoma In: Fletcher CDM, Unni KK, Mertens F (eds.) Pathology and Genetics of tumours of soft tissue and bone. World Health Organization Classification of Tumours, Lyon, IARC Press, France, pp: 255-256.

7. Shakked RJ, Geller DS, Gorlick R, Dorfman HD (2012) Mesenchymal chondrosarcoma: clinicopathologic study of 20 cases. Archives of Pathology and Laboratory Medicine 136: 61-75.

8. Chen JY, Hsu SS, Ho JT (2004) Extraskeletal intracranial mesenchymal chondrosarcoma: case report and literature review. Kaohsiung J Med Sci 20: $240-246$

9. De Padua M, Bhandari TP (2009) Primary mesenchymal chondrosarcoma of the breast. Indian J Pathol Microbiol 52: 129-130.

10. Font RL, Ray R, Mazow ML, Del Valle M (2009) Mesenchymal chondrosarcoma of the orbit: a unique radiologic-pathologic correlation. Ophthal Plast Reconstr Surg 25: 219-222.
11. Gomez-Brouchet A, Soulie M, Delisle MB, Escourrou G (2001) Mesenchymal chondrosarcoma of the kidney. J Urol 166: p. 2305.

12. Wehrli BM, Huang W, De Crombrugghe B, Ayala AG, Czerniak B (2003) Sox9, a master regulator of chondrogenesis, distinguishes mesenchymal chondrosarcoma from other small blue round cell tumors. Hum Pathol 34: 263-269.

13. Fanburg-Smith JC, Auerbach A, Marwaha JS, Wang Z, Rushing EJ (2010) Reappraisal of mesenchymal chondrosarcoma: novel morphologic observations of the hyaline cartilage and endochondral ossification and b-catenin, Sox9, and osteocalcin immunostaining of 22 cases. Hum Pathol 41: 653-662.

14. Panagopoulos I, Gorunova L, Bjerkehagen B, Boye K, Heim S (2014) Chromosome aberrations and HEY1-NCOA2 fusion gene in a mesenchymal chondrosarcoma. Oncology Reports 32: 40-44.

15. Cesari M, Bertoni F, Bacchini P, Mercuri M, Palmerini E, et al. (2007) Mesenchymal chondrosarcoma: an analysis of patients treated at a single institution. Tumori 93: 423-427.

16. Knott PD, Gannon FH, Thompson LD (2003) Mesenchymal chondrosarcoma of the sinonasal tract: a clinicopathological study of 13 cases with a review of the literature. Laryngoscope 113: 783-790.

17. Rushing EJ, Armonda RA, Ansari Q, Mena H (1996) Mesenchymal chondrosarcoma: a clinicopathologic and flow cytometric study of 13 cases presenting in the central nervous system. Cancer 77: 1884-1891.

18. Dantonello TM, Int-Veen C, Leuschner I (2008) Mesenchymal chondrosarcoma of soft tissues and bone in children, adolescents, and young adults: experiences of the CWS and COSS study groups. Cancer 112: 2424-2431.

19. Granter SR, Renshaw AA, Fletcher CD, Bhan AK, Rosenberg AE (1996) CD99 reactivity in mesenchymal chondrosarcoma. Hum Pathol 27: 1273-1276.

20. Ambros IM, Ambros PF, Strehl S, Kovar H, Gadner H, et al. (1991) MIC2 is a specific marker for Ewing's sarcoma and peripheral primitive neuroectodermal tumors. Evidence for a common histogenesis of Ewing's sarcoma and peripheral primitive neuroectodermal tumors from MIC2 expression and specific chromosome aberration. Cancer 67: 1886-1893.

21. Muller S, Soder S, Oliveira AM, Inwards CY, Aigner T (2005) Type II collagen as specific marker for mesenchymal chondrosarcomas compared to other small cell sarcomas of the skeleton. Mod Pathol 18: 1088-1094.

22. Lee AF, Hayes MM, LeBrun D (2011) FLI-1 distinguishes Ewing sarcoma from small cell osteosarcoma and mesenchymal chondrosarcoma. Appl Immunohistochem Mol Morphol 19: 233-238.

23. Sainati L, Scapinello A, Montaldi A (1993) A mesenchymal chondrosarcoma of a child with the reciprocal translocation $(11 ; 22)$ (q24;q12). Cancer Genet Cytogenet 71: 144-147.

24. Roberts P, Burchill SA, Brownhill S (2008) Ploidy and karyotype complexity are powerful prognostic indicators in the Ewing's sarcoma family of tumors: a study by the United Kingdom Cancer Cytogenetics and the Children's Cancer and Leukaemia Group. Genes Chromosomes Cancer 47: 207-220.

25. Wang L, Motoi T, Khanin R, Olshen A, Mertens F, et al. (2012) Identification of a novel, recurrent HEY1-NCOA2 fusion in mesenchymal chondrosarcoma based on a genome-wide screen of exonlevel expression data. Genes, Chromosomes and Cancer 51: 127-139.

26. Nakayama R, Miura Y, Ogino J, Susa M, Watanabe I, et al. (2012) Detection of HEY1-NCOA2 fusion by fluorescence in-situ hybridization in formalin-fixed paraffin-embedded tissues as a possible diagnostic tool for mesenchymal chondrosarcoma. Pathology international 62: 823-826.

27. Park YK, Park HR, Chi SG (2000) Overexpression of p53 and rare genetic mutation in mesenchymal chondrosarcoma. Oncol Rep 7: 1041-1047.

28. Brown RE, Boyle JL (2003) Mesenchymal chondrosarcoma: molecular characterization by a proteomic approach, with morphogenic and therapeutic implications. Ann Clin Lab Sci 33: 131-141.

29. Horner MJ, Ries LAG, Krapcho M (2002) SEER cancer statistics review: tumors of adolescents and young adults, 2002-2006. National Cancer Institute, Maryland. 